\title{
History, Current Status, and Future of Infrared Identification
}

\author{
Francine Prokoski, Ph.D. \\ Mikos Ltd. \\ mikos@gte.net
}

\begin{abstract}
Infrared identification (IRID) is a biometric which offers the security of fingerprints with the convenience of facial recognition. Twenty years after being first proposed, IRID systems are becoming marketable. During the intervening time, IR camera technology has improved significantly and price reductions have been dramatic. Further improvements in IR camera sensitivity, array size, and variable optics, as well as further price reductions, are coming, just as interest is growing in biometrics. Several approaches have been developed, tested, and evaluated to extract and analyze features from infrared images of the face and body. IRID has been to have significant advantages over other biometrics in certain applications --- not only in the traditional biometric security markets, but also in communications and health care.
\end{abstract}

\section{Introduction}

The general public has accepted the need for increased security measures. In fact they expect to see surveillance cameras in public areas, have learned to accept long waits for security checks at airports, and are accustomed to being asked for ID in order to cash checks, make withdrawals, obtain medical benefits, and pick up their children from day care. People are increasingly willing to pay for security by spending dollars and time, and by sacrificing some privacy. The market for security technology is therefore experiencing major growth.

Biometrics is the fastest growing segment of the security industry. Fingerprint readers, handwriting verification, facial recognition, hand geometry, retinal and iris scanners, DNA analysis, and voice recognition are all becoming familiar techniques for identifying who a person is, and for providing links into databases of personal information. For some applications, biometric identification is simply more convenient than remembering a password or carrying a keycard. However, for many applications accurate and rapid automatic identification is of serious importance.

\section{Background on IRID Technology}

The goal of developing infrared identification has been to develop a technique which is as unique as fingerprints and as convenient as facial recognition. The anatomical information which is utilized by IRID involves subsurface features unique to each person. Those features may be imaged at a distance, using passive infrared sensor technology, with or without the cooperation of the subject.. The thermal patterns seen in an IR image derive primarily from the pattern of superficial blood vessels which are those under the skin but above bone and muscle. The vessels transport warm blood throughout the body, and heat the skin above. Skin which is directly above a blood vessel is on the average $0.1^{\circ} \mathrm{C}$ warmer than adjacent skin. A typical human face displays an apparent temperature range of about $8^{\circ} \mathrm{C}$.

That range and sensitivity are well within the specifications of current IR detector technology. The diameter of a blood vessel is on the order of $0.1 "$, which is well within the resolution of current focal plane arrays and optics. The complexity and vastness of the layout of about 5 kilometers of blood vessels in the head and face assures that each person's vascular arrangement is irreproducible and hence unique. Even identical twins have different thermogram features. IRID provides the capability for rapid, on-the-fly positive identification, under all lighting conditions including total darkness.

Problems with visual facial recognition in dim lighting conditions are exacerbated when the subject has dark skin. The result is a difference in accuracy of performance as a function of skin color. A visual face recognition system which is optimized for identification of light-skinned persons could be prone to higher false alarms among darkskinned persons; a situation which is unacceptable. Infrared identification sacrifices color recognition for lighting independence, with the result that it provides color-blind identification with the same accuracy for everyone. When colors of hair, eyes, skin, clothing, etc. is essential to match eyewitness descriptions, a color band can be added to the camera to provide that information. Positive automated identification, however, should be performed from IR images. 
Fingerprint ridges are formed early in gestation, when the thin skin responds to the underlying developing vascular structure. Therefore both fingerprint features and facial thermogram features have their origin in the detailed network of blood vessels and finer structures of the cardiovascular system. A comparison between minutiae from facial thermograms and from fingerprints, based upon the anatomical structures underlying each, supports the experimental findings that facial thermograms are unique, as fingerprints have long been assumed to be unique for each person. For many biometric applications, identification based upon facial thermograms is preferable to use of fingerprints, since the former require no physical contact with the subject and can be collected on-the-fly.

While there is no way to prove that facial thermograms are unique, it is possible to show they contain inherently more vaiations than fingerprints. Similarly, it has never been proven that fingerprints are unique. However, that working hypothesis has been supported by many years of experience. Facial thermogram minutiae is analogous to fingerprint minutiae in that two sets of minutiae may be considered to identify the same person if a significant number of the minutiae in the two sets have corresponding positions and characteristics.

Classification of facial thermograms can be performed to partition a database and reduce the search for matching facial patterns, just as fingerprints may be classified as to type prior to searching a database. In the US, 16 minutiae points must correspond in order for an unknown fingerprint to be considered a match to a known print for evidentiary purposes. Efforts are underway to establish guidelines for similar evidentiary rules regarding the use of infrared facial imagery for positive identification in legal proceedings.

Depending on the IR camera used, and the imaging scenario, the quality of the resulting infrared imagery may not be sufficient to support extraction of minutiae. Other feature extraction techniques can be used which may provide sufficient accuracy in certain identification tasks.

\section{Experimental Variables}

Facial thermograms reflect variations in apparent temperatures across the facial surface which are produced by changes in ambient temperature and air flow conditions, as well as by exercise, metabolic activity associated with ingesting food and drink, illness, and drugs. Many other factors can produce localized changes. These include wearing tight clothing, being under stress, having a migraine headache, blushing, or having an infected tooth. The high level of variability in facial thermograms, representing the extreme level of sensitivity to a large number of variables, presents a challenge to find features which are persistent enough to use in automated identification.

The solution is to ignore the apparent temperature values and use only the anatomical features they reflect which are invariant to changes in physiological and ambient conditions. Consider this analogous to analyzing fingerprints; whether the print is formed by coating the finger with oil, blood, blue ink, or chocolate, and whether a rolled or latent print is seen, the same fingerprint details emerge. Similarly, facial thermograms yield the same blood vessel pathways and minutiae regardless of apparent temperatures.

Only a single frame of infrared video is required to uniquely identify a person if it is taken from the same aspect angle as a known reference image. IR images can be taken at the same time and with the same care as a standard photograph used for passports. There are strong correlations between the infrared and visual facial images: head shape and size, location and shape and size of features. The database of images can be segmented into classes using those values, and the same classification system will work for visual or infrared images.

\section{Feature Extraction from Thermal Images}

IRID integrates infrared imaging, anatomical modeling, pattern recognition, and image compression to achieve a biometric capability. The initial research approaches utilized thermal contours for identification, and matched them through template, fractal, or wavelet methods. That approach is tailored for cooperative access control applications in which the subject voluntarily interacts with the system, such as at an ATM or point of sales (POS) terminal. Its accuracy is affected by activities which disturb the edges of the contours. This includes eyeglasses being put on or off or changed. It also includes variations in head position relative to the camera, and changes in facial and cranial hair.

Several approaches to feature definition and extraction have been considered. Choice depends on the camera used, the level of subject cooperation, the throughput required, and the price to be paid.

\subsection{Historical Approaches}

Metrics Matching. Some current commercial face recognition systems use face metrics obtained from visual images. Those systems admittedly cannot differentiate identical twins, and are inaccurate when the lighting is dim or when it is not uniformly illuminating the face. Even when a face is well lit, differences in angle of view can affect the manual or automatic locating of feature points. Shadows, glint, makeup, and disguise can cause 
greater errors in locating the feature points and deriving relative distances. Only about a dozen feature points such as inner and outer corners of the eyes, top and bottom points where the ears are connected to the head, centroids of the nostrils, and lowermost tip of the nose can be precisely and repeatedly located if seen in the visual image. Many more feature points can be extracted from IR imagery, by using specific vascular references, such as the branching of particular blood vessels.

\section{Table 1 Range of Face Metrics in Sample}

Faces scaled to 100 pixels between pupils:

$\begin{array}{lrr}\text { inner eye corners } & 20 & 29 \\ \text { outer eye corners } & 141 & 178 \\ \text { nose base width } & 48 & 62 \\ \text { nosetip to mouth line } & 28 & 54 \\ \text { nose tip to face center } & 90 & 118 \\ \text { face width at nose base } & 178 & 215 \\ \text { face width at centerline } & 201 & 221 \\ \text { face width at mouth line } & 199 & 220 \\ \text { ear top(s) to face center } & 160 & 185 \\ \text { ear bottom(s) to face center } & 195 & 230 \\ \text { face height above center } & 122 & 136 \\ \text { face height below center } & 143 & 203\end{array}$

However, there is no large scale test, using either IR or visual imaging, indicating that any person's set of facial measurements is unique, and often only a few such measurements are available from imagery --- particularly in non-cooperative applications. Face metrics is therefore not considered a robust identification technique.

Table 1 presents 14 metrics which may be obtained from either IR or visual images. The face center is defined for this purpose as the midpoint between the eyes, located on the line connecting the centers of the pupils. A sample of 100 images in IR and the corresponding 100 visual images were manually analyzed to produce the ranges of measurements shown. All images were scaled to 100 pixels between the pupils. Such metrics can be applied to both visual and IR images as a classification technique to partition the respective databases in order to reduce search time. This can assist rapid comparison of faces-in-the-crowd streaming images against a Watch List of known faces. Partition boundaries need to have sufficient overlap to account for imprecision in feature designation and metric computation

Template Matching. If thermograms are standardized as to size, and histogram normalized, the areas about the canthi and the inner checks can be compared using template matching with rather good results. On databases of 250 people, a crossover error of $7.5 \%$ was obtained by matching three template areas, using older cooled IR cameras having NETD of approximately $0.1^{\circ} \mathrm{C}$. At that level of sensitivity, detailed thermal contours are not seen and so shape analysis does not apply. Inexpensive uncooled IR cameras can now provide that level of verification accuracy with a simple processor chip installed in the camera, for a system cost below $\$ 5000$.

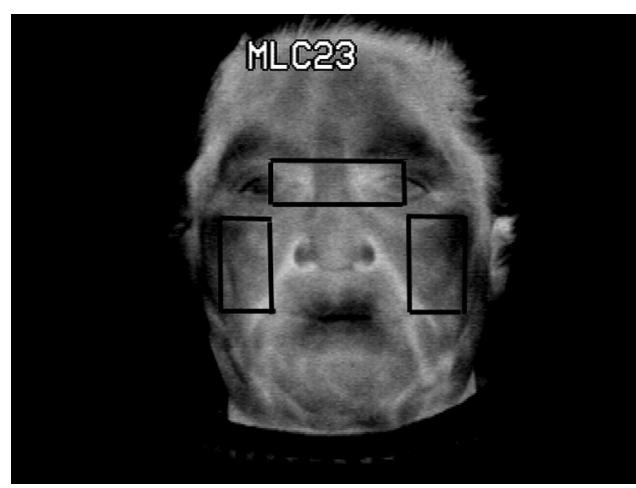

Figure 1 Face Templates

Elemental Shape Real-Time Matching. With IR cameras having NETD of $0.7^{\circ} \mathrm{C}$ or less, 100 or more different closed thermal contours are seen in each face. The sets of shapes are unique for each individual, even in the case of identical twins, because they result from the underlying complex network of veins and arteries. Variation in defining the thermal slices from one image to another has the effect of shrinking or enlarging the resulting shapes, while keeping the centroid location and other features of the shapes constant.

Each nesting of thermal closed contours is called an "elemental shape". Pre-production systems based on elemental shape analysis have been built and have undergone extensive testing with more then 250 persons, and databases of more than 10,000 images.. This has proven the persistence of those features of the facial thermograms which are used for identification and the uniqueness of each person's thermal image. Facial thermograms for a limited number of subjects have been obtained for periods of 8 to 23 years, demonstrating the required persistence over those durations. Automated IRID using elemental shapes in real time has achieved $96 \%$ accuracy for cooperative access control applications.. Studies on pairs of identical twins have demonstrated that they can be separately identified through elemental shape analysis.

Elemental Shape Post Matching. The totality of shapes in a library of facial thermal images were analyzed. Eigenshape analysis was used to compare characteristics of each shape including: perimeter, area, centroid $\mathrm{x}$ and $\mathrm{y}$ locations, minimum and maximum chord length through 
the centroid, standard deviation of that length, minimum and maximum chord length between perimeter points, standard deviation of that length, and area/perimeter. Shapes whose edges are interrupted may need to be ignored when compared against images with different edge effects. As examples: Shapes along the face edge will change when the face is turned. Shapes around the eyes will be affected when glasses are put on.

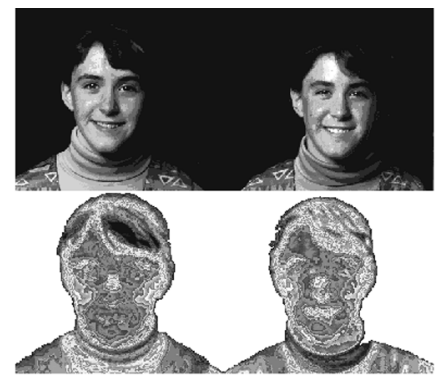

Figure 2 Thermal Contours of Identical Twins

A non-cooperative, non-real-time faces-in-the-crowd version of the IRID system was also built and demonstrated, with more than 100 persons represented in a database of 500 images. Twelve targeted persons were selected, and the database was searched for all appearances by the targeted persons. $98 \%$ accuracy was achieved, with no false positives. All the database images were manually selected from IR videotapes obtained at trade shows. Only in-focus, essentially full face images were selected and manually scaled and centered. Processing was not done in real time. The 11-coefficient eigenanalysis required 20 hours on a 486/66 PC.
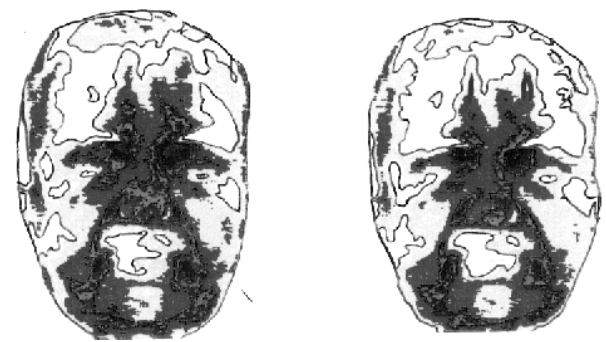

Figure 3 Variation in Thermal Contours with $10^{\circ} \mathrm{C}$ Change

Each person's image was characterized by a set of 11coefficient polynomials. The difference in eigenspace between any two images was calculated to yield a measurement to which a threshold was applied to make a "match / no match" decision. While those studies proved that IRID can produce high accuracy identification for noncooperative faces-in-the-crowd applications, the calculation techniques are computationally intensive and would need to be hardware-based for real-time use.

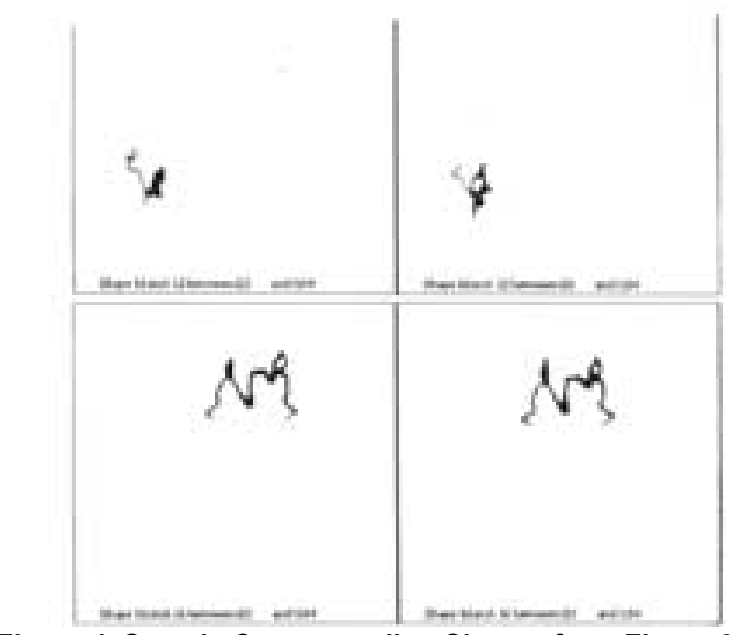

Figure 4 Sample Corresponding Shapes from Figure 3

Wavelet Analysis. Prototype IRID systems for access control were developed by Unisys Corporation under a licensing agreement. The configured systems were designed for unattended cooperative access control. Components included IR camera, Pentium I PC-class computer, face acquisition assembly, keypad, and the enrollment subsystem of a monitor and keyboard. The face acquisition assembly included the mechanisms which move the camera and focus it so that the face is within the field of view. This was the first large scale IRID test to use an uncooled camera --- a BST $320 \times 240$ built by Lockheed Martin.

A proprietary wavelet analysis and matching engine from Fast Pattern Recognition was used. Performance exceeded $98.5 \%$ accuracy including persons who wore eyeglasses some or all of the time. However, when persons switched glasses, the system error increased appreciably. This error mode needs to be eliminated and the cost of the hardware matching engine needs to be drastically reduced from the $\$ 25,000$ figure before this approach to IRID can be successfully marketed.

\subsection{Recent and Current Research}

IRID systems now under development will utilize lower cost cooled and uncooled infrared cameras, and will perform identification based upon recognition of anatomical structures rather than thermal contours. As the demand for biometric identification systems grows, and as implementations are considered for very large populations, such as national registries and airline passengers, there are related needs for data compaction, rapid comparisons, and adaptable decision levels to meet variable requirements for security and throughput. In response, alternative analysis techniques are under 
development which take advantage of improved IR camera imagery.

Symmetry Waveforms. Ideally, each human face is bilaterally symmetrical when viewed in either visual or IR imagery. However, no one's face is truly symmetrical in either spectrum. As camera sensitivity and resolution improve, more subtle asymmetries can be seen. Starting with the hypothesis that the details of each person's asymmetries are unique to that person, a number of analysis techniques have been developed to exploit those individual variations for identification purposes.

Six techniques have been investigated. In each case the image is first scaled and histogram normalized. In each case only a vertical swath of the face within the outer corners of the eyes is analyzed, in order to minimize the effects of head rotation. Therefore, these techniques are applicable only to imagery in which both eyes can be seen. Each technique assigns a value to each horizontal line of pixels in the face swath. These values constitute the symmetry waveform.

The first three techniques analyze the grey scale image and produce a waveform which represents: 1) the total grey scale value to either side of the vertical line bisecting the swath, 2) the x-value at which the total grey values within the swath to either side are equal, 3) the foldover point which provides the smallest cumulative difference. between grey scale values to either side of the point.

The fourth technique analyzes thermal contours and locates the balance point between all the contours which cross that horizontal --- with each crossing point given equal weight. The fifth technique is similar but analyzes the vasculature by binarizing but not eroding the image. Larger blood vessels contribute more pixels to the balance calculation.

The sixth technique considers minutiae symmetry, finding the best balance point among minutiae along that horizontal. Due to the relatively small number of minutiae, and the various causes of imprecision in specifying their location, each minutia is represented as a dot which is several pixels in diameter. The size is a function of the expected location error .

Symmetry waveforms can be used for tracking of head rotation, tilt, and tip, and to provide accurate alignment of images. The obtained symmetry waveforms from the latest project are highly encouraging for the subjects considered (23). Each of the six techniques produced unique and repeatable waveforms for the small subject pool used. The results were obtained from an Amber Radiance InSb 256x256 camera with $12.5 \mathrm{~mm}$ lens. The specified NETD was $0.025^{\circ} \mathrm{C}$. Symmetry analysis is not expected to provide an identification technique suitable for large database, but may be adequate for small databases or for classification purposes.

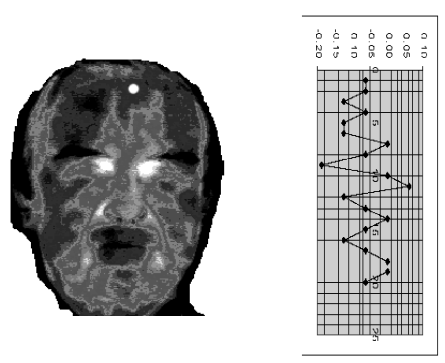

Figure 5 Symmetry Waveform

Face Codes. It may be possible to derive an unique facecode repeatedly from each person, eliminating the need for comparing against a database Based upon the degree of variation seen in different persons' symmetry waveforms, a coding approach was considered which would derive a one-dimensional bar code from a symmetry waveform. Although it seemed straightforward to designate a bar code line for each significant transition in the waveform, it is in fact a sophisticated calculation that is required. Additional improvement is needed in developing related algorithms.

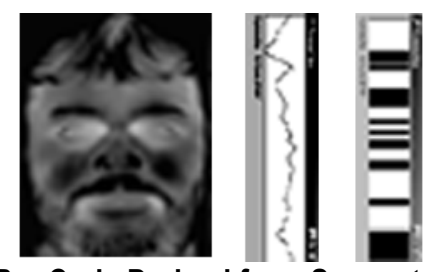

Figure 6 Bar Code Derived from Symmetry Waveform

Two-dimensional bar coding of the face has been considered, in which the face is divided into cells and each cell is compared to a library of face segments. The best matching segment is selected and its code used for that cell. Matching is done using FlashCorrelation $\AA$. While this approach appears promising, further work is needed to establish an optimized library, which should be generated by considering a large database of facial mages from the particular camera to be used in a system.

A simple binary code can be generated by dividing the standardized face center into cells and indicating those in which minutiae are present.

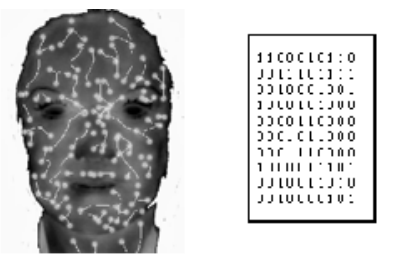

Figure 7 2-D Bar Code from Facial Minutiae 
More sophisticated encodings also consider the type of minutiae and interconnections. For the databases used to date, which typically included 5000 images of 500 persons, the results from face encoding did not appear to be any more useful that simply downsampling an image that has been deskewed, centered, scaled, and histogram normalized. Resulting thumbnail images that are 32 pixels wide provide accurate positive identification with minimal processing required.
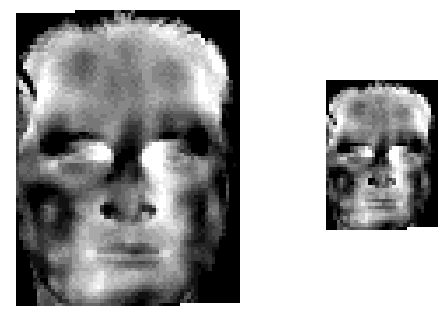

Figure 8 IR Thumbnail Retains Identifiable Features

It is expected that an effective thermal face encoding technique will eventually be developed. The coding scheme will take account of head position, and allow for degraded accuracy of identification when only a partial face is seen. Resulting systems will scale up to very large population. Depending on the computational complexity of the processing required, such a capability could allow for rapid throughput with minimal cooperation by the entrant. This technique would also support real time faces-in-the-crowd and digital signature applications.

Thermal vs Visual Minutiae. Another IRID approach focuses on the correlation between IR and visual facial imagery. That capability broadens the security market to include uses where no reference database of thermal images exists. Existing databases of visual images (mug shots, DMV photos, passport photos, company IDs, surveillance camera images) can be automatically matched against images from an IR surveillance camera. The most dramatic application is for automated screening at airports for known terrorists. However, the same system and techniques apply to any surveillance usage; for security at government buildings, courthouses, embassies, foreign corporate offices, and so forth. Although several companies offer facial ID systems which operate with visual imagery alone and claim to provide similar capabilities, they cannot perform accurately when the lighting is dim or when disguises are worn.

The IR/visual correlation capability extends the markets for IRID beyond security into communication process control applications. In particular, use of a dualband IR/Visual camera enables significant bandwidth compression of "talking head" video. A system user or communications service provider can achieve high compression ratios by transmitting only a baseline visual facial image, followed by movement vectors for those thermal minutiae which are involved in changes of expression or speech. The animated face is then reconstructed at the receiving end. Applications for this technology include: videotelephone, video email, videoconferencing, news broadcasts, and electronic commerce. Together, these applications represent a sizable and growing percentage of the overall transmission of internet and telephone messages.

IR/Visual correlation also extends the markets for IRID to health care. There is a growing market for home diagnostic devices, and infrared cameras linked to diagnostic software are destined to become as common as thermometers in the home medicine cabinet. We anticipate that routine infrared monitoring of elderly patients on heart and arthritis medications, and postsurgical care of wounds or infections will be among the first applications for home-based diagnostic on-line systems. Household accidents, including falls and burns and insect bites, will also be imaged with a home infrared camera which transmits live video to an HMO diagnostic center for realtime advice and preadmission approval to emergency centers. Future diagnostic uses for infrared and for hyperspectral imaging will be tremendously varied; in homes, nursing homes, clinics, hospitals, ambulances, police cruisers, gymnasiums, and basically anywhere there is currently a first aid kit. Those applications, however, are outside the scope of this paper, which deals only with the identification aspects of infrared imaging.

Identification is, however, an important aspect of medical care. It includes issues of patient privacy and who has access to patient data. It includes assuring that the proper patient receives the proper treatment. It includes procedures for computerized patient recordkeeping. It also includes the issue of insurance and medicare fraud. Those issues can all be addressed by several different security approaches, including other biometrics. In every case the basics are the same: each person is positively identified prior to any service being performed, or any access granted. There is another medical identification requirement, however, for which IRID is uniquely applicable.

The same cardiovascular network, including blood vessels and other elements of the vascular and lymph systems, which permeates the face area is similarly distributed throughout the body. This provides the capability for identifying any body part just as it provides for identifying the face. More importantly, it provides a network of approximately 2400 minutiae whose locations and interconnections can repeatedly be determined passively and from a remote imager. These 2400 points serve as anchor points to facilitate morphing an image to a standard image, or to another image of the same type from 
the same patient. Since infrared cameras are passive, and are becoming inexpensive compared to other medical imaging devices such as a sonogram or X-ray, an IR camera can be used in conjunction with other imaging systems at minimal additional cost. This provides the capability to annotate the IR minutiae onto other types of medical images; thereby facilitating sensor fusion among a variety of diagnostic medical system.

An even simpler application is the automated comparison of same-modality images taken at different times. The analysis system would determine whether a particular medical condition was improving or static or regressing. The thermal minutiae would be used for automatic alignment of the two images, which could have been taken using different imaging systems, which produce different resolution images, from different distances and angles, and with different areas of view. With the collection of a large volume of annotated imagery and associated patient histories, we predict that automated libraries will be generated. A given patient's current medical images could then be automatically compared not only with his own earlier images, but also to standardized images representing composites formed from other patients' data in the library. That would enable automatic determination for example that not only is a surgical patient improving since his last image was taken; but that with a confidence level of $95 \%$ his progress is most similar to a patient group which had a $90 \%$ survival rate ten years after the same surgery.

Perhaps one of the good outcomes of managed health care will be the opportunity to better manage health care information. Use of the Standardized Infrared Minuties Coordinate System (SIMCOS) technique is expected to facilitate improvements in medical informatics, by providing a structure for development of standardized image libraries and automated comparison techniques.

\subsection{Anticipated Performance of Future IRID Systems}

The cameras used during the twenty year development of IRID included a variety of detector materials and cooling techniques, array sizes, and optics with different resulting resolution and sensitivity. However, there has been a steady decline in prices and improvement in performance. The decline was noticeably accelerated by DARPA's funding during the 1990's, especially with respect to enhanced performance and uncooled detector technology. Uncooled IRID systems within the next five years are expected to have greater thermal sensitivity, better resolution, faster autofocus, auto gain control, expanded depth of focus, and prices below $\$ 5000$ in quantities. Multispectral video/mid-IR/far-IR imagers are also expected to be commercially available. Cooperative IRID access control systems will achieve the costs and accuracy of current live scan fingerprint readers, but will offer non-contact passive operation and will apply to all persons without exception. Applications to computer security, ATM and point of sales terminals, entry control systems, and long-range smart surveillance will offer the ease of photographs with the security of fingerprints at a cost competitive with other high performance biometrics.

Low-cost miniature IR/Visual cameras are expected to become available within the next five years to support computer security, secure videoconferencing, talking head video compression, synthetic face animation, and health care applications. They will support FaceCode derivation to provide digital signature authentication of identity onto real time transmissions, and will facilitate the introduction of electronic voting and enhance the security of ecommerce.

Dual-mode "look at / look for" IRID systems are envisioned which will first identify an authorized user and then provide him with access and IR viewing capability. Such systems would be deployed on weapons, in vehicles, and associated with remote surveillance operations. They provide dual use of a single IR imager, thereby offering reduced overall weight and cost.

\section{Areas of Application for IRID}

Research to date has investigated the various IR feature extraction and analysis techniques for human identification in five application areas.

\subsection{Access Control.}

IRID systems for access control aim at insuring the identity and authorization of persons seeking entry to physical locations or in transactions for which only an initial identification is required. The IRID system can be stand-alone or be integrated into other systems.

Prototype IRID systems for access control were developed by Unisys Corporation under a licensing agreement. The configured systems were designed for unattended cooperative access control. Components included IR camera, Pentium I PC-class computer, face acquisition assembly, keypad, and the enrollment subsystem of a monitor and keyboard. The face acquisition assembly includes the mechanisms which move the camera and focus it so that the subject's face is within the field of view. The primary cost component of the system is the infrared camera. The camera in the current system was designed and built by Lockheed Martin for Unisys. It uses an uncooled BST array and produces images of $320 \times 240$ pixels in the 8-12 micron band. The stand-alone access control system version of the IRID system was designed to sell for $\$ 25,000$, and to offer a false positive rate of less than $1 \%$ and false negative rate of less than $2 \%$ for single access 
attempts. Improved systems now under development will incorporate various hardware, software, and procedural modifications. which are expected to improve system accuracy while reducing the price.

\subsection{Computer Security.}

IRID offers exceptional features for real-time computer security. IRID continually re-verifies the identity of the system user, and confirms his authorization level prior to each action he performs. The continuity verification uses the patented FlashCorrelation ${ }^{\circledR}$ technique which permits normal head and body movements within the field of view of the camera. However, the system automatically blanks the screen, locks the keyboard and peripheral devices if the user moves out of the field of view of the camera, or does not look at the monitor or interact with the system for a programmable period of time.

The IRID computer security system provides positive continuous biometric identification of persons logging-on and interacting with protected networks. The infrared vascular pattern provides a digital signature which can be used to tag files created or accessed. Once logged-on, the system does not interfere with the user other than to control activities based upon prior authorizations.

Onlookers can be detected and their presence used to blank the screen and otherwise temporarily disable the system. Movement of the user out of the field of view of the IR camera also blanks the screen and temporarily disables the keyboard and peripherals. Return to within the field of view automatically re-enables the system, although prolonged absence will cause automatic log-off.

\subsection{Smart Surveillance.}

IR imaging has significant advantages for "smart" surveillance, which has been coined to refer to automatically screening persons seen in surveillance operations against a watch list of persons who are to receive special attention. The watch list will generally include persons who are to be denied access to a facility, or whose presence is to be reported to authorities. However, a watch list could also contain persons who are to be given preferential treatment or access, such as undercover police.

The IRID smart surveillance systems will extend the usage of IR surveillance to automated identification of persons whose IR facial image has not been previously collected, but whose visible facial image is available. These systems will utilize coincident features which appear in both imaging modes plus anatomical features seen in the IR image. IRID Smart Surveillance Systems will provide the ability to automatically surveil an area in the dark, find each face within the scanned area, and match it against a Watch List of visible images.

Recurring offenders and locations with recurring criminal incidents are prime candidates for installation of Smart Surveillance systems. Parole or probation of repeat offenders can include prohibitions against visiting hot spot areas. Smart Surveillance technology provides the means to verify compliance in a cost-effect way. Counterterrorism will be aided by the ability to automatically check each person entering a monitored location such as an airport, customs area, or courthouse against a Watch List of known or suspected terrorists.

Crime prevention and crime solving can also be aided by the automated comparison of IR facial images collected at different locations or at different times even when the subjects are not included in any current Watch List. The fact that an unknown person is visiting certain sites or a particular sequence of sites, or that he appears on-site immediately following an incident, or that he is seen to meet with persons on a Watch List may provide important linkage data in predicting criminal activity or correctly assigning responsibility after it occurs.

\subsection{Talking Head Video Compression.}

Change in facial expression and the action of speech cause movements in affected areas of the face, particularly the lips but also the eye, chin, forehead, and cheek areas. Encoding of facial expressions and facial movements during speech is currently being studied for bandwidth reduction in the transmission of "talking head" video for applications such as videophone, videoconferencing, video email, synthetic speech, and face animation. The intent is to transmit a baseline image followed by encoded changes to that image, with reconstitution of the animated face at the receiving end. This process offers significant bandwidth reduction, but may produce imagery in which the talking face seems stiff and unnatural or does not appear to be synchronized with the audio, giving the unacceptable look of a dubbed foreign film.

All previous studies involved modeling the facial movements based upon the relocation of certain observable points of the face, such as the corners of the mouth. The various models differ in the extent to which they consider the underlying facial muscles and nerves. There are few observable reference points on a generalized face, especially under uncontrolled lighting conditions. In particular, there are no observable reference points in the cheek areas, and none in the forehead area except possibly skin creases. When the talking head is that of a dark skinned person, the reconstructed image may show further degradation of subtle facial changes. 
Use of a dualband IR/Visual camera at the transmission end offers the potential for marked improvements. Infrared minutiae are more numerous than visible markers and are present throughout the face, including areas of the cheeks and forehead and chin where no visible minutiae may be present. Therefore, modeling of the movements of infrared minutiae can provide finer detailed replication of expressions and speech than modeling based upon visual references.

By processing sequences of images taken from known expressions and/or known speech elements, a sequence of movements of infrared minutiae can be extracted which correspond to that expression or speech element for that person or for persons in general. Subsequently, when the same sequence of movements of infrared minutiae is seen, it can be inferred that the person is displaying the same expression or speech element as during the initial sequence. This enables the automated determination of expression or speech, allowing for compression of transmitted video in conjunction with audio. The combination may offer additional composite compression and improved synchronization. The same basic technique can also be used to create a dictionary of facial expressions and speech elements for use in animation of a synthetic face.

\subsection{Health Care.}

Increasing use of medical imaging techniques is producing an enormous amount of medical history data for each patient. Medical diagnosis and treatment increasingly involves imaging from various sensor modalities, such as: x-ray, CAT scan, MRI, sonograms, and mammograms. As a person grows older, moves, changes doctors, experiences various medical events, changes employer and insurance company and perhaps his or her name, the task of maintaining a complete and accurate medical history becomes increasingly complex. The volume of information per patient, coupled with the desire to reduce medical costs, has led to a goal of computerized patient records. The growing use of HMOs and telemedicine requires methods by which physicians can rapidly review and assess computerized medical records containing image data and written comments generated by various medical personnel on patients they may have never seen.

A primary requirement in establishing computerized records is for a method of automated patient identification. It is essential that the correct diagnosis and treatment are performed on the correct patient. This includes emergency patients who are unconscious, mentally incompetent, intoxicated, or otherwise unable to identify themselves. All computerized medical data must be correctly annotated with the patient's ID and filed in the correct patient record. Automated identification is particularly important in large databases where they may be multiple persons with the same name, and in telemedicine applications where the patient, his medical records, the primary and consulting physicians may all be in different locations. Aside from accidental errors associated with human and system mistakes, there are intentional errors created for reasons of insurance fraud, in which imagery or other data submitted with claims is not from the person represented, or in which multiple claims are filed under multiple identities for the same person. The need exists to improve the accuracy of associating medical imagery and other data with the identity of the person from whom it was derived.

A primary requirement for medical data exploitation is for automated comparison of images taken from the same individual, but at different times, under different conditions, and perhaps using different sensors. This type of sensor fusion can utilize a standard set of reference points on the human body which will accommodate changes in age, weight and health status. Since the reference points may need to be frequently determined, their collection must pose no risk to the individual. A technique is needed which annotates standard reference and registration points on any and all medical imaging modalities, and which can be used to standardize medical library files in order to compare different imagery from the same person or from different persons.

Work is proceeding on a Standardized Infrared Minutiae Correlation System (SIMCOS) for annotation of standardized thermal minutiae onto medical images. The minutiae serve to identify the patient, align different images, and unlock access into the patient's computerized files. The SIMCOS approach is analogous to the use of fingerprints for automated identification of persons; however, it utilizes all or any part of the body's surface, and requires no contact or cooperation with the patient. Therefore, it is particularly suited for dealing with noncooperative patients such as the aged, infants, unconscious, mentally handicapped, or drug and alcohol impaired. Minutiae patterns can serve as a security access code for opening the specific patient's electronic files.

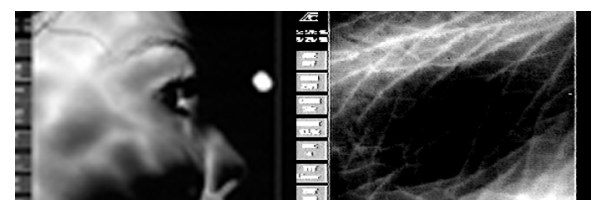

Figure 9 Vasculature seen in IR Imagery

Current commercial IR cameras do not have the resolution to provide direct viewing of vasculature over the entire face in a single frame. However, as Figure 9 
indicates, partial face views are highly detailed and can be mosaiced to cover the entire face or other body area. A microscope lens allows for viewing the specific influence of an underlying blood vessel on the skin and hair above. Future IR camera developments for medical applications may renew interest in linear array imagers. It is expected that hyperspectral linear arrays will be developed for whole-body scanning. The same minutiae extraction and SIMCOS standardization technique will be applicable to the resulting imagery.

Standardizing database images facilitates comparison of imagery during growth from childhood to adulthood, compilation of medical libraries of images from large numbers of people, and automated comparisons of current imagery against the vast libraries for diagnostic purposes. Standardization to a common set of thermal minutiae would also provide a common reference for comparing images obtained from different sensors which produce different resolution images. There are many potential applications for medical image fusion, and for automated positioning of patients relative to diagnostic, surgical, and treatment equipment. One example follows.

Landmarks on nuclear medicine studies. Certain studies, such as for myocardial perfusion, require two or more studies be performed on a given patient at two different times. Precise repositioning is difficult due to the many relevant factors which are not known in advance, such as orientation of the heart within the body and size of the various portions of the heart.

The requirement for repositioning may therefore lead to successive manual repositionings of the camera and patient in order to repeat as closely as possible the earlier study. Using SIMCOS, standardized thermal minutiae can be used for universal positioning of all patients, or for specific repetition of a given patient's positioning. The thermal minutiae can be annotated on the resulting imagery. The SIMCOS technique is expected to be more precise and repeatable than current techniques for identifying the long axis of the left ventricle from nuclear medicine SPECT data.

\section{Other Future Applications}

The systems described to this point have all been installed on fixed or mobile platforms from which they identified whatever person was within their field of view. There are also significant applications for miniature IRID systems which would be integrated into worn computer systems and dedicated to watching the single specific wearer. Design of the bodyworn IRID system would likely involve a Bluetooth-compliant communications capability to securely broadcast in a limited range the identity and status of the wearer.
Transmitted information could include the wearer's level of attention, point of attention, level of fatigue, indications of stress, facial expression, and whether or not he is talking. This information is derived from a combination of thermal minutiae micromovement analysis and monitoring of such classic observables as blink rate and eye fixation patterns, sweat, and head nod.

System design involves using a bodyworn computer, with the remote sensor head of an IR camera mounted along with a microphone on a headset. The rest of the IR camera would be worn along with a computer on a belt or backpack. Initial systems would continually, or on demand, broadcast the identity of the person. They would also monitor and transmit his attention, stress, and fatigue levels. Those are all capabilities which are within the capability of current IR imagers and algorithms but have not yet been prototyped.

Future systems would use IR facial monitoring to enhance speech recognition, particularly for use in noisy environments. They would also incorporate features to perk-up the wearer's attention, and require additional confirmation of activities initiated when the wearer's stress or fatigue levels were above threshold. Incorporating these additional features will require extensive further research.

\section{References}

[1] F. J. Prokoski and R. Riedel, Infrared Identification of Faces and Body Parts; chapter 9 in BIOMETRICS: Personal Identification in Networked Society", edited by Sharatchandra Pankanti, IBM and Kluwer Academic Publishers, 1998.

[2] F. J. Prokoski, Patient ID and Registration of Medical Images using SIMCOS; MEDTEC, Medical Technology Conference of the National Security Industrial Association, Orlando, Florida, 9-11 July 1996.

[3] F. J. Prokoski, NC-TEST; Non-Contact Thermal Emissions Screening Technique for Drug and Alcohol Detection, SPIE Conference on New Law Enforcement Technologies, 18-22 November 1996, Boston.

[4] F. J. Prokoski, Use of Low-Cost IR Imagers for Emergency Response: Medical, Fire, and Law Enforcement; Tutorial Presentation by 1995 Fifth Annual IEEE Dual Use Technologies and Applications Conference, Utica NY, May 1995

[5] F. J. Prokoski., IDENTAS; Identification from All Sources, 1995 Fifth Annual IEEE Dual Use Technologies and Applications Conference, Utica NY, May 1995

[6] F. J. Prokoski., Facial Identification from Infrared Imagery, 1994 Fourth Annual IEEE Dual Use Technologies and Applications Conference, Utica NY, May 1994

FlashCorrelation ${ }^{\circledR}$ is a registered trademark of Mikos Ltd. 